Sharif University of Technology
Scientia Iranica
Transactions E: Industrial Engineering
http://scientiairanica.sharif.edu

\title{
Type-2 fuzzy rule-based expert system for diagnosis of spinal cord disorders
}

\author{
S. Rahimi Damirchi-Darasi ${ }^{a}$, M.H. Fazel Zarandi ${ }^{a, b, *}$, I.B. Turksen ${ }^{b, c}$, and \\ M. Izadi ${ }^{\mathrm{d}}$
}

a. Department of Industrial Engineering, Amirkabir University of Technology, Tehran, Iran.

b. Knowledge Intelligent System Laboratory, University of Toronto, Toronto, Canada.

c. Department of Industrial Engineering, TOBB University of Economics and Technology, Ankara, Turkey.

d. Fayyazbakhsh and Erfan Hospitals, Tehran, Iran.

Received 6 December 2015; received in revised form 11 July 2017; accepted 5 March 2018

\author{
KEYWORDS \\ Type-2 fuzzy expert \\ system; \\ Forward-backward \\ chaining; \\ Parameter \\ optimization; \\ Spinal cord disorder; \\ Rule-based expert \\ system.
}

\begin{abstract}
The majority of people have experienced pain in their low back or neck in their lives. In this paper, a type-2 fuzzy rule-based expert system is presented for diagnosing the spinal cord disorders. The interval type-2 fuzzy logic system permits us to handle the high uncertainty of diagnosing the type of disorder and its severity. The spinal cord disorders are studied in five categories using historical data and clinical symptoms of the patients. The main novelty of this paper lies in presentation of the interval type2 fuzzy hybrid rule-based system, which is a combination of the forward and backward chaining approaches in its inference engine and avoids unnecessary medical questions. Use of parametric operations for fuzzy calculations increases the robustness of the system and the compatibility of the diagnosis with a wide range of physicians' diagnosis. The outputs of the system are comprised of type of disorder, location, and severity as well as the necessity of taking an M.R. Image. A comparison of the performance of the developed system with the expert shows an acceptable accuracy of the system in diagnosing the disorders and determining the necessity of the M.R. Image.

(C) 2019 Sharif University of Technology. All rights reserved.
\end{abstract}

\section{Introduction}

According to the statistical information of W.H.O., low back pain is ranked the second among the most probable physical problems and nearly $80 \%$ of people experience it in their lives. Neck pain is another spinal cord disorder in which more than $30 \%$ of people have been involved [1]. Spinal cord disorders diagnosis is based on a synthesis of history, clinical examina-

*. Corresponding author. Tel.: +982164545378

E-mail addresses: saeede.rahimi@aut.ac.ir (S. Rahimi

Damirchi-Darasi); zarandi@aut.ac.ir (M.H. Fazel Zarandi);

turksen@mie.utoronto.ca (I.B.Turksen);

mina.borujeni@gmail.com (M. Izadi)

doi: $10.24200 /$ sci. 2018.20228 tion, and paraclinical testing, like MRI. According to Ambulatory Health Care Data, more than 20 million MRI tests are conducted annually in the United States and $50 \%$ of them are performed because of the spine problems. In recent years, the shortage of diagnostic radiologists has been a concern [2]. Computer aided diagnostic systems play a vital role by helping the physicians to perform a better diagnosis $[3,4]$.

Many studies have developed new methods of diagnosing herniated disc, as one of the common spinal disorders, based on MRI and/or CT; however, more than $90 \%$ of the patients with low back pain do not need to take MRI for diagnosing the problem and/or investigating the MRI does not change the treatment methods. Medical philosophy, vague boundaries of symptoms, and diagnosis require using the framework 
of fuzzy sets, systems, and relations to model the medical expert system [5]. Malaria [6], viral hepatitis [7], and cardiovascular disease [8] are the first diseases for which fuzzy methods have been used to model expert systems. In recent years, Fazel Zarandi et al. [9] used a fuzzy rule based expert system for diagnosing asthma. Kadhim et al. [10] developed a fuzzy expert system for diagnosing low back pain based on clinical observation symptoms using fuzzy rules. Sari et al. [11] developed two expert systems, namely artificial neural network and adaptive neuro-fuzzy inference system, to assess the low back pain level. Esteban et al. [12] developed a fuzzy linguistic web system in which personalized exercise or recommendations were offered for prevention. Gulbandilar et al. [13] constructed a fuzzy logic algorithm to identify low back pain intensity by using data of 169 patients. A fuzzy expert system was developed by Ohri et al. [14] to diagnose breast cancer. Gal et al. [15] proposed a fuzzy expert system to predict subchondral sclerosis. In the study of Katigari et al. [16], a fuzzy expert system was presented to diagnose diabetic neuropathy. Their system was constructed by using 244 medical records.

In some situations, in which uncertainty of data and the degree of vagueness of information are too high, type- 2 fuzzy may perform better in modelling. Among the studies on type- 2 fuzzy medical expert systems, Fazel Zarandi et al. [17] used type-2 fuzzy methods of image processing for diagnosing the brain tumor. Rahimi Damirchi-Darasi et al. [18] developed an expert system to diagnose degenerative disc disease based on type- 2 fuzzy methods. They showed that the high uncertainty of some clinical symptoms required more accuracy to get acceptable results. Zarinbal et al. [19, 20] developed a type- 2 fuzzy image processing expert system to diagnose brain tumors. They evaluated the system performance using 95 MRI scans, showing good capacity of diagnosis.

The aim of this paper is to develop a fuzzy rule based expert system to achieve six objectives:

- Handling the high uncertainty of clinical variables;

- Combining forward chaining with backward chaining based on a direct approach in designing the architecture of the inference engine;

- Optimizing the parameters of fuzzy membership function based on different diagnoses of physicians and increasing the robustness of the proposed system;

- Diagnosing a wide variety of spinal cord disorders as well as type and location of the disorder;

- Declaring the necessity of MRI and severity of the disorders.

The rest of the paper is organized as follows: Section 2 reviews the type-2 fuzzy sets and systems and presents the definitions of the most common spinal cord disorders. The methodology of the system is presented in Section 3. Structure and the inference mechanism of the system are discussed in Section 4. Section 5 thoroughly explains the structure of each module of the knowledge base. Evaluation of the system performance is carried out in Section 6. Finally, the discussion and conclusion are presented in Section 7 .

\section{Background}

\subsection{Spinal cord disorders}

Due to overlapping of the disorders with each other, the most important issue in diagnosing the spinal cord disorders is classifying them. Fazel Zarandi et al. [21] categorized spinal cord disorders of the patients that visited the physician in five groups: Mechanical pain; herniated disc; spinal stenosis; spinal deformity like scoliosis, lordosis, or kyphosis; and red flag. There is a definition for each disorder: Mechanical pain refers to any type of back pain caused by placing abnormal stress on muscles of the vertebral column [22]; herniated disc refers to a problem with one of the rubbery cushions (discs) between the individual bones (vertebrae) that are stacked up to make the spine [23]; spinal stenosis is narrowing of the open spaces within the spine, which can put pressure on the spinal cord and the nerves that travel through the spine; and red flag is when the patients have some emergency symptoms and the physician applies results of paraclinical testing, immediately.

Each of the disorders mostly occurs in a specific region. Spinal stenosis generally occurs in the neck and lower back [24]. Approximately, $90 \%$ of herniated discs occur in the low back at disc L4/5 and disc L5$\mathrm{S} 1$ and cause pain in the $\mathrm{L} 5$ or $\mathrm{S} 1$ nerve that radiates down the sciatic nerve [25]. The most common discs in the cervical spine to herniate are disc $\mathrm{C} 5 / 6$ and disc $\mathrm{C} 6 / 7$. The next most common is disc $\mathrm{C} 4 / 5$ and disc C7-T1 may rarely be herniated [26]. Figure 1 represents the relationship between spinal nerve roots and vertebrae [27].

In medical terminology, risk factors are the factors that increase the potential for back and neck problems, and yellow flag symptoms [28] are the factors that highlight the risk of chronicity in the patients.

Overlapping of the disorders with each other and the existing different ways to present the pain in body make diagnosis of the disorder and assessment of its severity difficult. The proposed expert system is the extension of the study by Rahimi Damirchi-Darasi et al. [18] and it investigates the clinical symptoms of the patients as well as risk factors in diagnosing all the five groups of disorders with type-2 fuzzy logic system to handle the uncertainties of vagueness in the clinical symptoms. 
Bone notch at the base of the neck is $\mathrm{C} 7$.

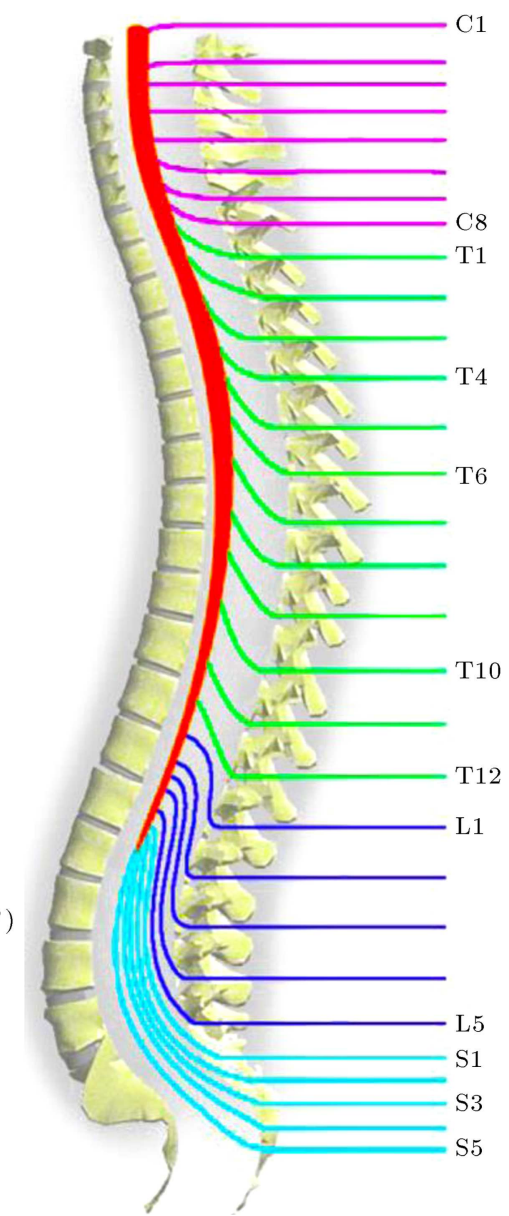

Cervical spinal nerve roots $\mathrm{C} 1-\mathrm{C} 7$ correspond with upper aspects of vertebral bodies.

Sensation of $\mathrm{C} 7$ nerve is for the middle finger

C8 and lower spinal nerve roots leave below the corresponding vertebral body

Sensation of T4 spinal nerve is approximately level with the nipple line.

Sensation of T6 spinal nerve root is approximately level with the bottom of the sternum.

Sensation of T10 spinal nerve root is approximately level with the abdomen.

Sensation of T12 spinal nerve root is approximately level with the public bone

The sensations of lumbar nerves are over the legs

Sensation of S3, S4 \& S5 nerves is the Perineal (genital) area.

Th coccygeal vertebrae are fused to make the

Figure 1. Diagram showing the relationship between spinal nerve roots and vertebrae [27].

\subsection{Type-2 Fuzzy Logic Systems (T2FLS)}

There are some sources of uncertainties in type-1 FLSs [29]. To handle them, Mendel and John [29] presented Type-2 fuzzy logic system. In this part of the paper, the structure of T2 FLS is presented.

A general T2 FLS is illustrated in Figure 2. If the antecedent and consequent sets in rules are type-2, the FLS is type-2. The major structural difference between T1 FLS and T2 FLS is that the defuzzifier block of
T1 FS is replaced by the output-processing block in T2 FLS. This block consists of type-reduction followed by defuzzification [30]. In the following subsections, the important terminology in developing the proposed expert system is explained.

\subsubsection{Approximate Reasoning (AR)}

Logical approximate reasoning and Mamdani approximate reasoning are two different methods used in inference engine of expert systems. The method of rea-

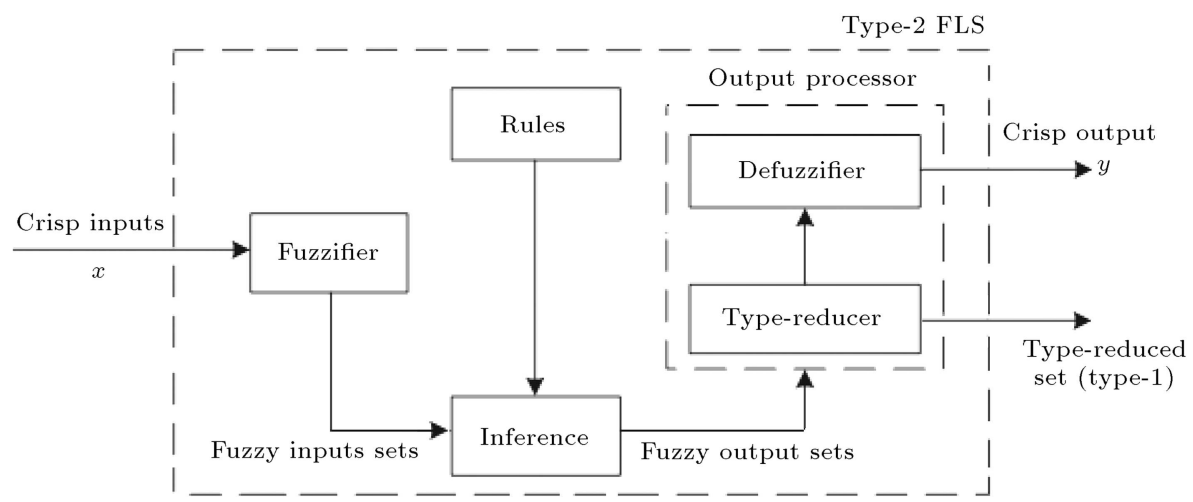

Figure 2. Type-2 FLS [29]. 
soning implemented in developing the proposed expert system is unified fuzzy reasoning. The unified fuzzy reasoning method is defined by logical approximate reasoning and Mamdani approximate reasoning [31].

Consider $\mu_{F_{L}}(y)$ as a fuzzy output of logical AR and $\mu_{F_{M}}(y)$ as a fuzzy output of Mamdani AR; the unified fuzzy reasoning method is defined as Eq. (1):

$$
\mu_{F}(y)=\beta \times \mu_{F_{L}}(y)+(1-\beta) \times \mu_{F_{M}}(y),
$$

where $\beta$ is the parameter of hybridization of logical approximate reasoning and Mamdani approximate reasoning.

\subsubsection{Type reduction}

As shown in Figure 2, the type-2 outputs of the inference engine must be processed by the output processor after its first operation, which is type reduction. Some methods of type reduction are centroid, center-ofsums, height, modified height, and center-of-sets [32]. Karnik and Mendel [33] and Karnik et al. [34] presented the details of centroid, height, center-of-sets, modified height, and center-of-sums type reductions. We use height type reduction method in this paper.

Also, $\mu_{\bar{B}^{l}}\left(\bar{y}^{l}\right)$ is the membership function of each point in interval type- 2 fuzzy sets and $h_{l}$ is height type reducer. If the domain of each $\mu_{\bar{B}^{l}}\left(\bar{y}^{l}\right)$ is represented by $\left[L_{l}, R_{l}\right]$, then $h_{l}=\left(L_{l}+R_{l}\right) / 2$.

\subsubsection{Defuzzifying}

The defuzzification of the type-reduced set is done to get a crisp output form of the type- 2 FLS. Leekwiick and Kerre [35] classified the most widely used defuzzification techniques into different groups. In this study, we use Yager parametric defuzzification. In Eq. (2), $y^{*}$ is defined as Yager parametric deffuzification [31].

$$
y^{*}(x)=\frac{\int_{y_{0}}^{y_{1}} y\left[\mu_{F}(y)\right]^{\alpha} d y}{\int_{y_{0}}^{y_{1}}\left[\mu_{F}(y)\right]^{\alpha} d y}, \quad \alpha>0 .
$$

\subsubsection{Operation on type-2 fuzzy sets}

Membership grades of type-2 sets are type-1 sets; therefore, we should be able to perform t-conorm and t-norm operations between type-1 sets. Fuzzy operations like complement, intersection, and union do not have unique operations, and they are contextdependent [31]. Here, the Yager classes of operations, which are used in developing the system, are defined as:

(a) The Yager class of fuzzy complements [32] is defined by Eq. (3):

$$
C(a)=\left(1-a^{\omega}\right)^{\frac{1}{\omega}}, \quad \omega>0 .
$$

(b) The class of Yager t-norm $(t)$, i.e., the intersection of $a, b$ [32], is defined by Eq. (4):

$$
\begin{aligned}
& t(a, b)=1-\min \left(1,\left[(1-a)^{\omega}+(1-b)^{\omega}\right]^{\frac{1}{\omega}}\right), \\
& \omega>0 .
\end{aligned}
$$

(c) The class of Yager t-conorm $(s)$, i.e., the union of $a, b$ [32], is defined by Eq. (5):

$$
s(a, b)=\min \left(1,\left[a^{\omega}+b^{\omega}\right]^{\frac{1}{\omega}}, \quad \omega>0 .\right.
$$

\section{Methodology}

Identifying the proposed expert system is performed based on a direct approach. The wide varieties of disorders, insufficiency, and imprecision of the patients' records require using a systemic approach to develop a more efficient system. The methodology of generating the proposed system is as follows:

- Identifying system inputs and outputs;

- Classifying the input variables;

- Identifying the knowledge base structure;

- Generating the knowledge base rules;

- Identifying inference mechanism of the system;

- Tuning the parameters of the system.

\subsection{Identifying system inputs and outputs}

The first step in system modelling is identification of the inputs and outputs. Due to the wide variety of disorders, the patients' perception about the disorders has a crucial role in diagnosing them. On the other hand, the perceptions have a vague nature. In order to attain comprehensive knowledge, 384 dialogs between different patients and the neurosurgeon are recorded. Identifying the inputs and outputs is done by negotiation with the expert, studying the problem domain, and using $50 \%$ of the data.

\subsection{Classifying the input variables}

Figure 3 presents the semantic network of symptoms and shows the most important input variables in cause and effect classes based on their nature and roles in diagnosing spinal cord disorders.

Cause variables are responsible for spinal cord disorders. Historical data form four classes, namely patients' perception, emergency problem symptoms (red flag symptoms), psychological problem symptoms (yellow flag symptoms), and risk factors. Clinical data consists in five classes of records, namely inspection, palpation, precaution, auscultation, and manipulation. The importance of the clinical symptoms varies with different disorders and the neurosurgeon emphasizes the most important factors.

By classifying the patients' primary perception based on expert knowledge, the four main questions 


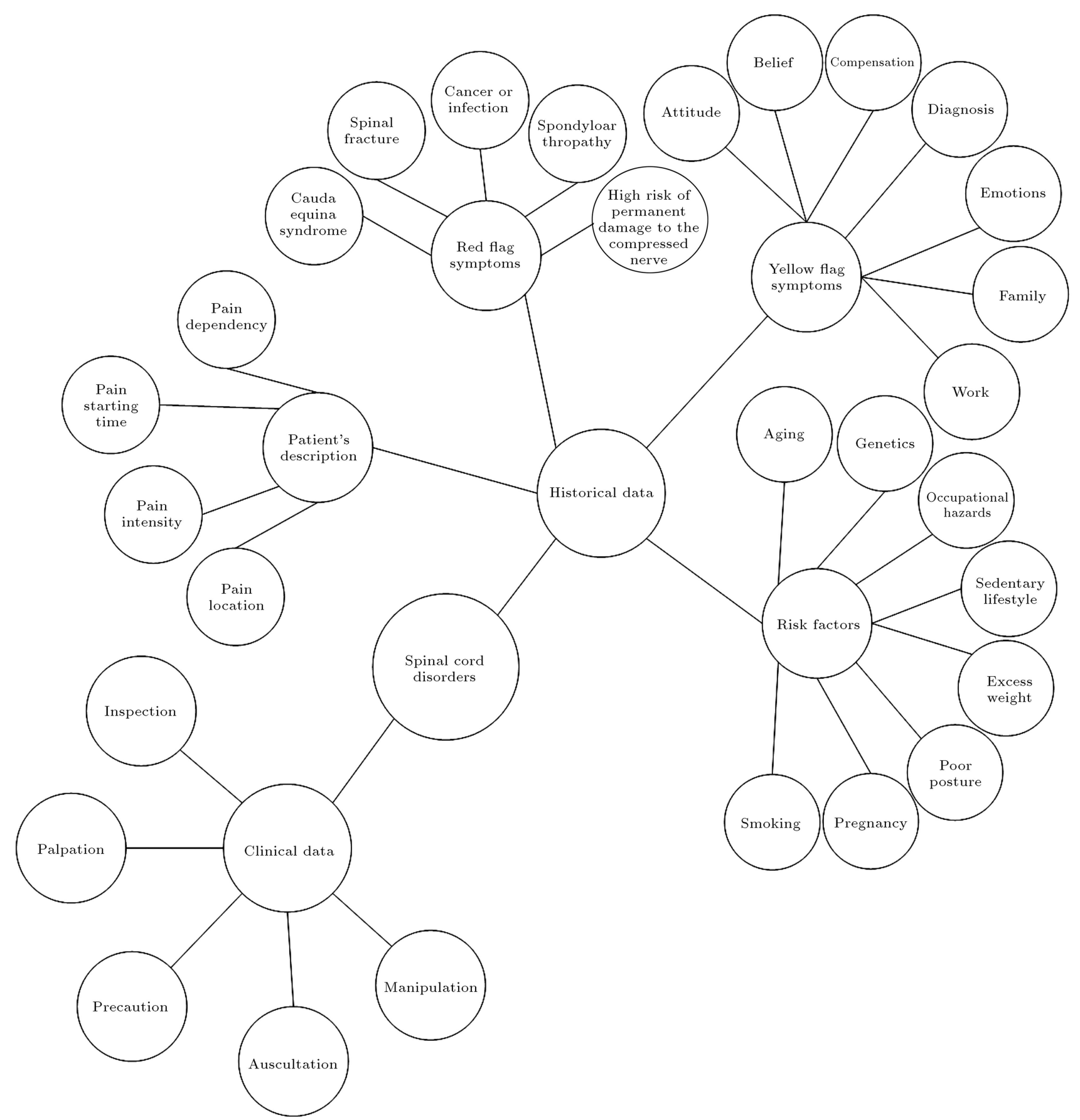

Figure 3. Semantic network of symptoms in diagnosing spinal cord disorders.

extracted are related to pain location, intensity and quality of the pain, the starting time of pain, and the dependency of pain on some position. Red flag symptoms [36] are categorized in five emergency problems: cauda equina, spinal fracture, cancer or infection, spondyloarthropathy, and high risk of permanent damage to the compressed nerve. Yellow flag symptoms [28] identify the psychosocial factors which highlight the patient's risk of chronicity and are categorized in seven factors: attitude, belief, compensation, diagnosis, emotions, family, and work. The main risk factors are aging, genetics, occupational hazards, lifestyle, weight, posture, pregnancy, and smoking [37].

\subsection{Identifying knowledge base structure}

As mentioned before, the neurosurgeons diagnose spinal cord disorder based on three types of data: historical, clinical, and paraclinical, like MRI. Historical and clinical data have a deterministic role in diagnosing the disorders and the necessity of providing the MRI is determined after investigating them. The proposed system uses historical and clinical data to 


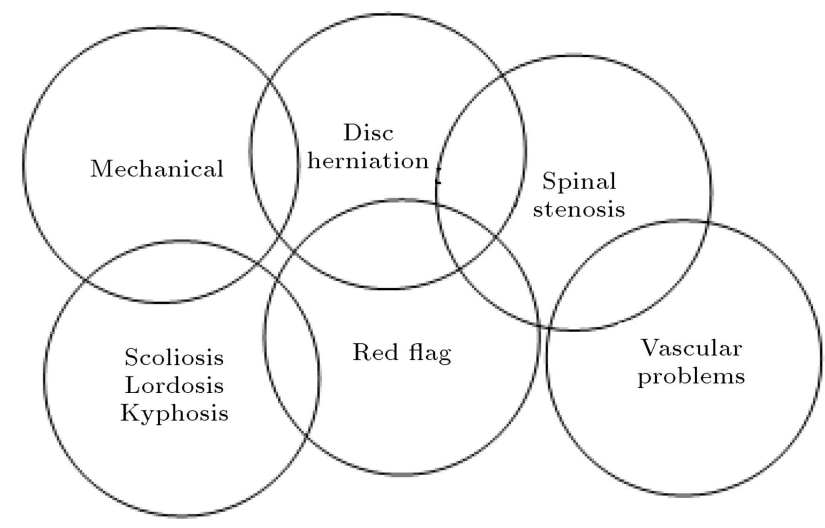

Figure 4. Overlapping between the spinal cord disorders.

diagnose location, type, and severity of the problem as well as to determine the necessity of providing MRI. The neurosurgeons do some clinical tests based on the result of investigation of the historical data. The overlap between disorders in some symptoms makes the diagnosis complicated. The overlapping is both between two spinal cord disorders and between spinal cord disorders with other problems like vascular problems. By classifying the symptoms, the main overlap between the disorders is modeled as in Figure 4. The overlap between disorders is fundamental for proposing the modular structure of the system.

Each of the disorders has its own clinical testing. To handle the complexity of the overlapping of the symptoms, the system investigates the historical data and decides what clinical testing is required for implementation. The identified input variables are organized in the modules based on their nature and relation with other variables.

\subsection{Generating knowledge base rules}

Based on perception of the patients and diagnosis of the neurosurgeon, the rules of the system using identified variables are extracted. The rules are categorized based on disorders and their overlapping. Finally, the neurosurgeon performs the final amendment. Because of the differences in the types of identified variables, their roles in diagnosis, and the importance of disorder diagnosis, the generated rules consist in three classes:

1. The variables with crisp (yes/no) values;

2. The variables with linguistic values, like severity of pulse, straightness of vertebrae, etc.;

3. The variables with linguistic variables having high uncertainty compared to variables of the second class, like severity of numbing, tingling, etc.

To handle the different degrees of uncertainty in variables, the system uses type-1 fuzzy logic in generating rules of the second-class and type- 2 fuzzy in generating the rules of the third class. In order to define the rules, Yager classes of intersection, union, and complement are assigned to the fuzzy operations.

\subsection{Identifying parameters of uncertain variables}

Two types of uncertainty are considered in developing the system: uncertainty in relations and uncertainty in values of the variables. Due to the high overlapping of the disorders and high uncertainty in the symptoms, defining the exact values for start and end points of disorders as well as the symptoms with linguistic variables is not possible. In order to define the intervals of the variables, Gaussian membership functions are assigned to the antecedents and consequences. Gaussian membership functions are defined by uncertain standard deviation and certain mean.

Consider $m_{k}^{j}$ as a certain means of Gaussian membership function and an uncertain standard deviation that takes value within $\left[\sigma_{k 1}^{j}, \sigma_{k 2}^{j}\right]$ [38], i.e., Eq. (6):

$$
\mu_{k}^{j}\left(x_{k}\right)=\exp \left[-\frac{1}{2}\left(\frac{x_{k}-m_{k}^{j}}{\sigma_{k}^{j}}\right)\right]^{2}, \sigma_{k}^{j}=\left[\sigma_{k 1}^{j}, \sigma_{k 2}^{j}\right] \text {. }
$$

This leads to the following definitions in Eq. (7) and Eq. (8) [38]:

$$
\begin{aligned}
& \bar{\mu}_{k}^{j}\left(x_{k}\right)=N\left(m_{k}^{j}, \sigma_{k 2}^{j} ; x_{k}\right), \\
& \underline{\mu}_{k}^{j}\left(x_{k}\right)=N\left(m_{k}^{j}, \sigma_{k 1}^{j} ; x_{k}\right),
\end{aligned}
$$

where, $\bar{\mu}_{k}^{j}\left(x_{k}\right)$ is the upper membership function, $\underline{\mu}_{k}^{j}\left(x_{k}\right)$ is the lower membership function, and for example, $N\left(m_{k}^{j}, \sigma_{k 1}^{j} ; x_{k}\right)$ is defined as Eq. (9):

$$
N\left(m_{k}^{j}, \sigma_{k 1}^{j} ; x_{k}\right) \triangleq \exp \left[-\frac{1}{2}\left(\frac{x_{k}-m_{k}^{j}}{\sigma_{k}^{j}}\right)\right]^{2},
$$

where, $k=1,2, \ldots, p$ and $j=1,2, \ldots, M$. " $p$ " shows the number of antecedents, " $M$ " indicates the number of rules, and $N$ is a Gaussian membership function of $m_{k}^{j}, \sigma_{k}^{j}, x_{k}$ [38].

\section{Structure and inference mechanism of the system}

Seventy-seven variables for diagnosing spinal cord disorders are identified, of which some are common in some disorders and others are specific to a special disorder. By modelling the method of the neurosurgeon in diagnosing the disorders, to avoid unnecessary questioning, the inference mechanism is hybrid of forward chaining and backward chaining. The system starts with the forward chaining phase to investigate some of the historical symptoms and makes a primal diagnosis by type reduction and defuzzification. The backward chaining phase tries to make more accurate diagnosis by investigating some of the clinical symptoms. 


\subsection{Knowledge base modulating}

To handle the high number of common variables between disorders, the knowledge base of the system has a modular structure. Inference mechanisms of modules of red flag, yellow flag, risk factor, herniated disc, mechanical pain, and spinal stenosis are forward chaining and inference mechanisms of modules of nerve roots, scoliosis lordosis kyphosis, and vascular problems are backward chaining; they will be explained in the following.

\subsection{Inference engine of the system}

To handle the different variables and symptoms, the hybrid of forward-backward chaining is proposed in the inference engine. Figures 5 and 8 contain flowcharts of the algorithm of the proposed system. A sequence of the modules is based on the symptoms' necessity and type of overlapping of the disorders.

\subsubsection{Forward chaining}

Figure 5 represents the forward chaining phase of the inference.

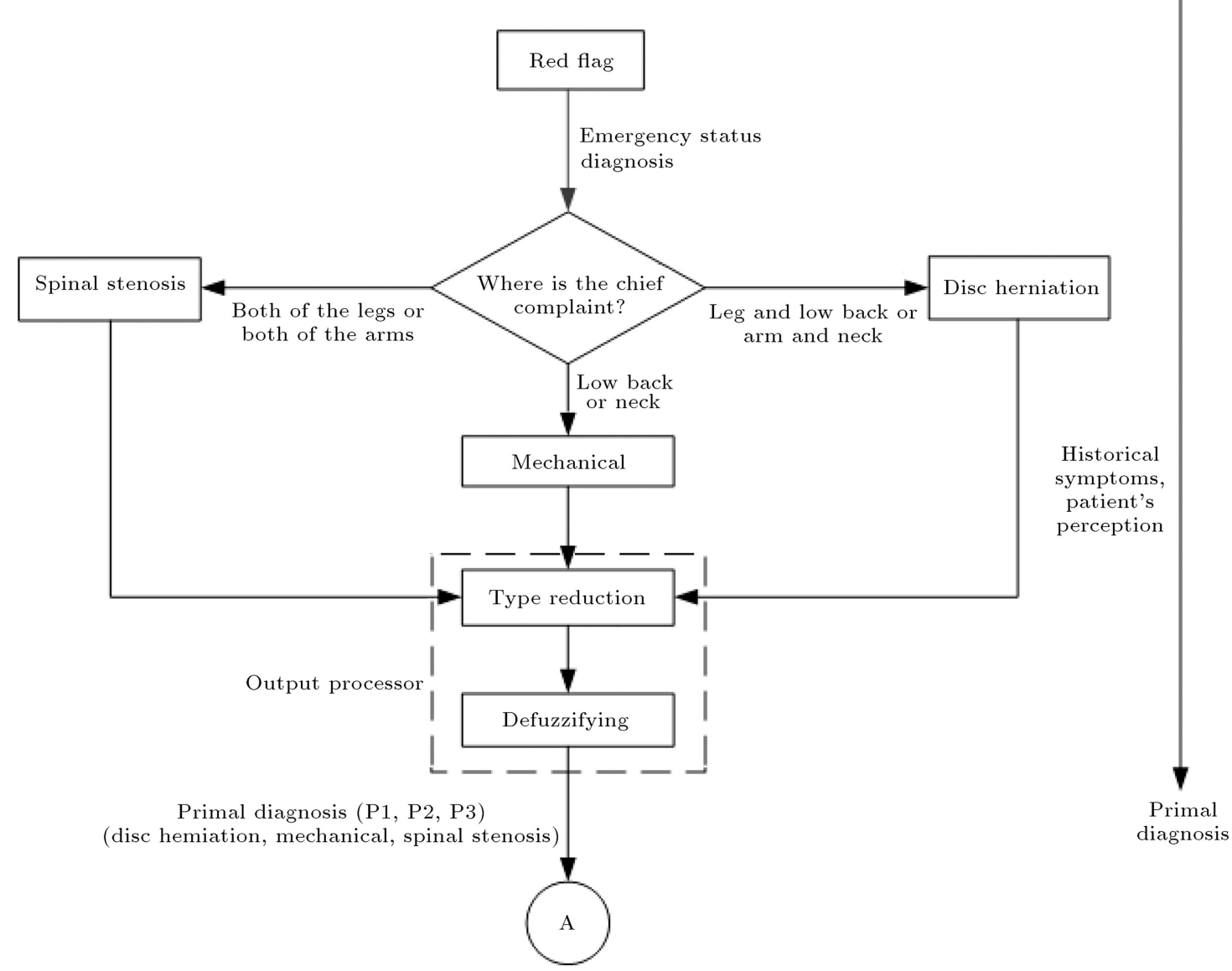

Figure 5. Algorithm of inference engine for diagnosing spinal cord disorders (forward chaining phase).

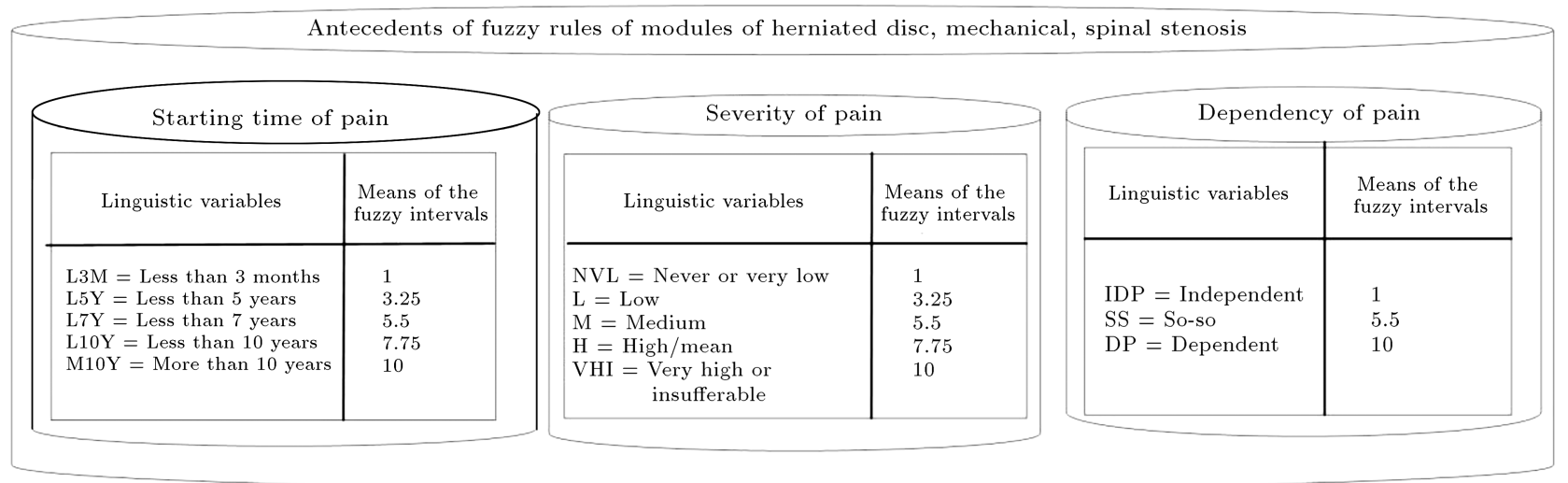

Figure 6. Antecedents of fuzzy rules of modules of herniated disc, mechanical pain, and spinal stenosis. 


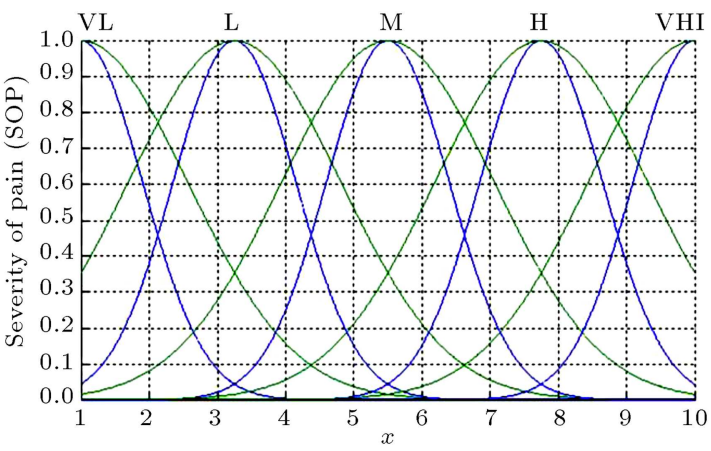

(a)

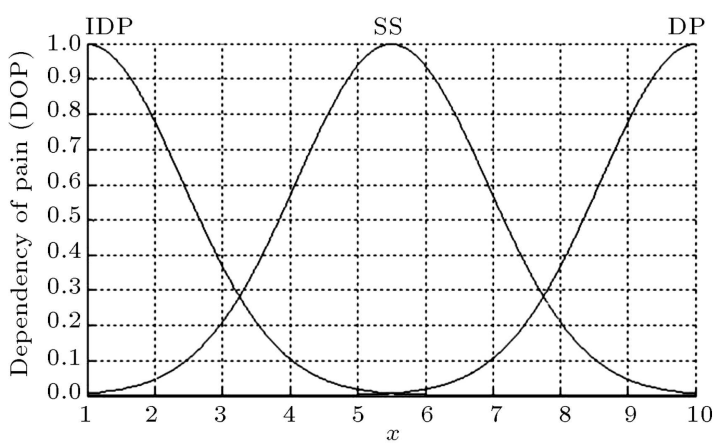

(c)

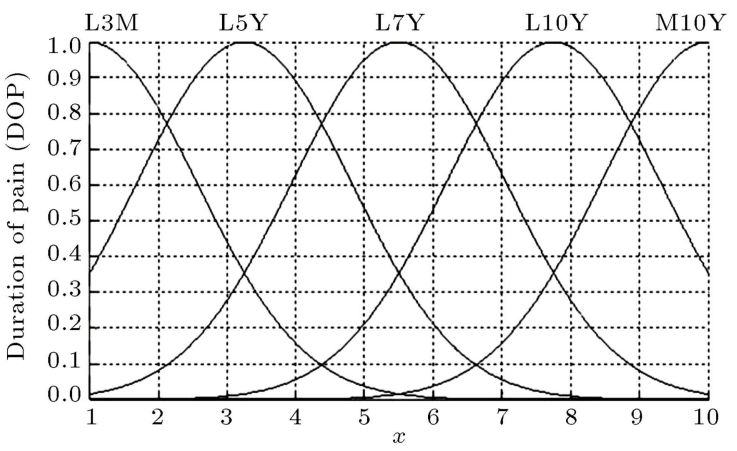

(b)

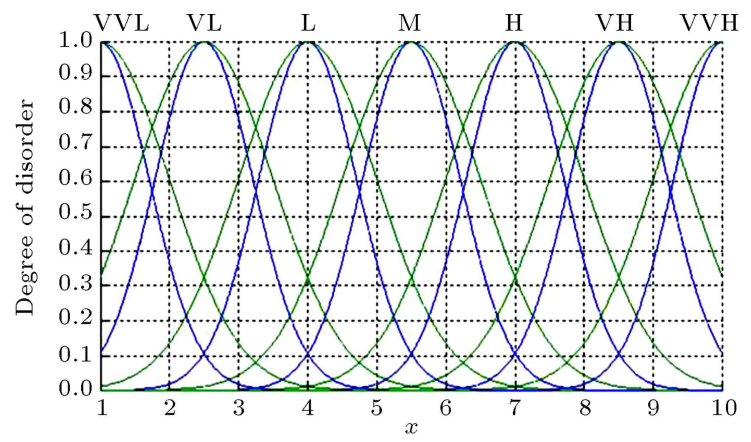

(d)

Figure 7. Membership functions: (a) Severity of pain, (b) starting time of pain, (c) dependency of pain, (d) degrees of disorders of herniated disc, mechanical pain, and spinal stenosis.

It starts the investigation by activating the module of the red flag to diagnose emergency patients, immediately. The output of this module declares emergency status of the patient. As represented in Figure 4, the central overlapping is between three main disorders: mechanical pain, herniated disc, and spinal stenosis. In the second step of the investigation, the system tries to diagnose between these three disorders. By asking about the patient's chief complaint, the system activates the specific module to get the patient's perception about the disorder and investigates them based on its knowledge base. If the chief complaint is pain in the leg and low back or arm and neck, the knowledge base of the module of herniated disc is activated; if the pain in the low back or in the neck is the chief complaint, the knowledge base of the module of mechanical pain is activated; and if the chief complaint is pain in both legs or both arms, the system activates the knowledge base of the module of spinal stenosis. The knowledge base of each of the modules consists in the rules and questions about severity of pain in the specific location, the starting time of pain, and the dependency of pain on some conditions. These variables have inherent uncertainty, which are represented in Figure 6. Figure 7(a), (b), and (c) depict the membership functions of these categories.

Consequences of the rules of the knowledge base of the herniated disc, mechanical pain, and spinal stenosis modules contain multiple outputs. The out- puts demonstrate the diagnosis of the three respective disorders. Figure 7 (d) shows the membership function of expert's diagnostic values of the three disorders. The rules of each module are explained in the structure of the modules. In order to have type- 1 outputs, the centroid method is assigned to the type- 2 outputs as the type reduction, and Yager defuzzifer is used to defuzzify them. The method used in the inference is the unified fuzzy reasoning. To obtain more robustness, the system tunes the parameters by optimizing the Root Mean Square Error (RMSE) function that is explained in Section 4.3.

\subsubsection{Backward chaining}

By defuzzifying the outputs of the module of the first stage, three numbers are achieved and the first stage in the inference engine (forward chaining phases) is finished. The system enters the second stage in the inference engine. The flowchart of the backward chaining phase is represented in Figure 8.

As shown in Figure 8, the system tries to investigate some clinical symptoms to prove the primal diagnosis. The maximum value among the three primal diagnoses specifies the direction to select the next module. If the value of herniated disc disorder is maximum, the system activates the module of the nerve root to assure itself of the diagnosis, and find the compressed nerve root and exact location of the abnormal disc. If the maximum value is for mechanical disorder, 


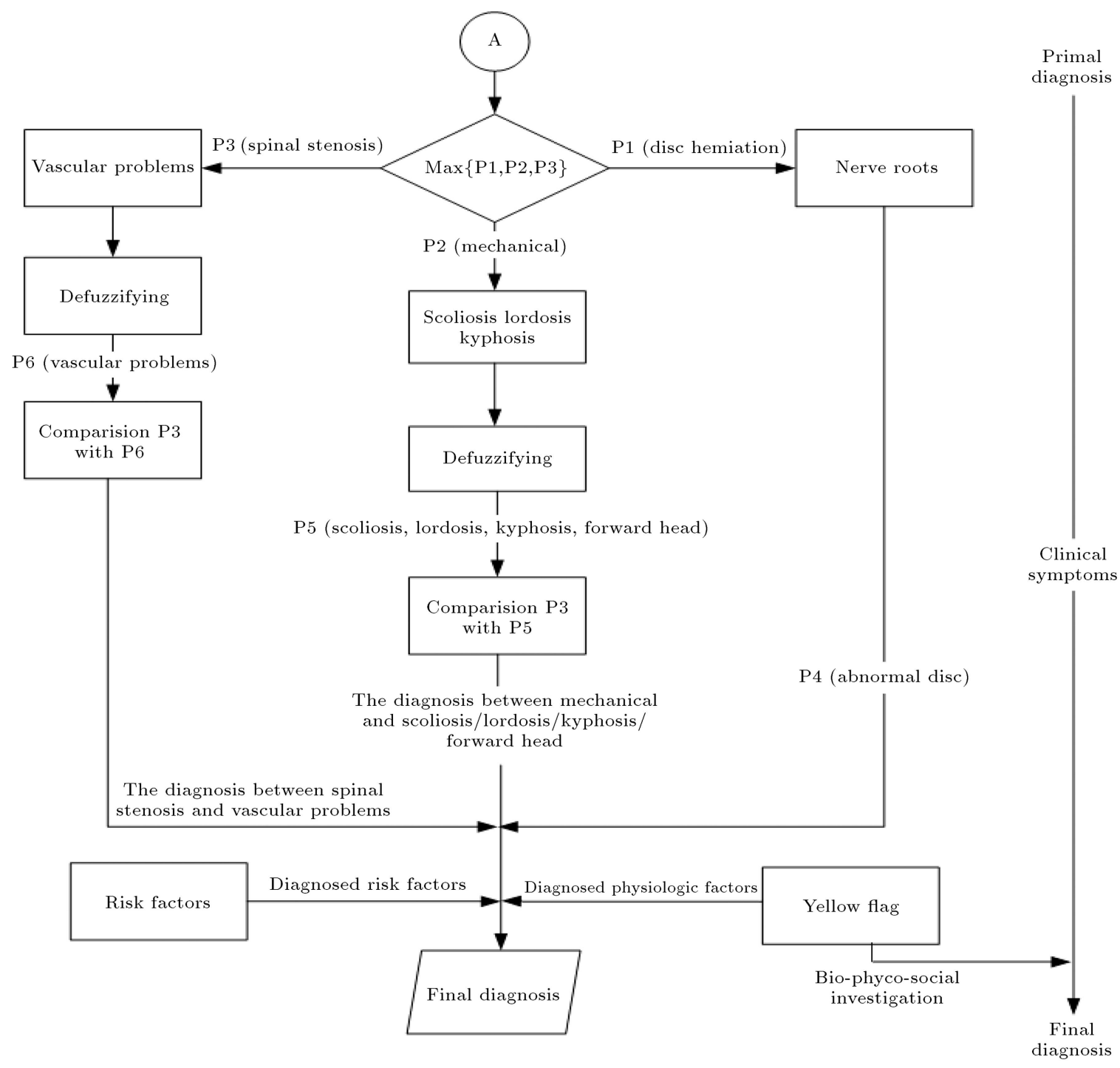

Figure 8. Algorithm of inference engine for diagnosing spinal cord disorders (backward chaining phase).

the system activates the module of scoliosis lordosis kyphosis for the diagnosis between the mechanical problem and scoliosis, lordosis, and kyphosis disorders. The module of the vascular problem is activated if the value of spinal stenosis disorder is maximum.

The type- 1 outputs of the inference engine must be processed next by the defuzzifier. The crisp output of this phase is compared with the crisp output of the first phase to diagnose between the disorders. The final investigation of the patient's symptoms is related to the risk factors and psychological problems. These two groups of symptoms are not the cause of the spinal cord disorders, but they can intensify them. Each of the modules of this phase are explained in Section 5. Final outputs of the system consist of (i) type of patient's disorder, (ii) exact location of abnormal disc in the low back or neck, (iii) declaring the necessity of MRI in four levels, and (iv) list of factors that intensify the disorder.

\subsection{Training}

The developed expert system has two main features in training: (i) Ability to adapt itself to different physicians and (ii) Ability to train itself to diagnose future patients more accurately, which are explained in the following. Due to the high overlap between the symptoms, different physicians may have different diagnoses regarding the same patients. In order to assimilate the expert system with diagnoses of different physicians, the system needs to be adaptive. Using parametric operations and functions could give this ability to the system. By using $25 \%$ of patients' records, the proposed system tunes its parameters by optimizing the error function presented in Eq. (10), and by updating the parameters after each correct diagnosis, the system could train itself. $Y$ is the primal diagnosis of the system and $\hat{Y}$ is the physician's primal diagnosis. $p, q, N$ are the parameters of t-norm, snorm, and negation, respectively. $\alpha$ is the parameter of 
Yager defuzzifier, $\beta$ is the parameter of hybridation of Mamdani and Logical inferences, and $n$ is the number of the patients considered to tune the parameters.

$$
\operatorname{RMSE}(p, q, N, \alpha, \beta)=\frac{1}{2} \sqrt{\frac{\sum_{i=1}^{n}\left(Y_{i}-\hat{Y}\right)\left(Y_{i}-\hat{Y}\right)^{T}}{n}} .
$$

\section{Structure of modules}

To explain the developed system specifically, the structure of the modules, their variables, inference engine mechanism, and the outputs are explained completely in this section.

\subsection{Module of red flag}

The task of module of red flag is immediate diagnosis of emergency patients. This module, which investigates emergency symptoms of the patient, are represented in Figure 9.

The inputs of this module are linguistic variables: never or very low, medium, very high or always. The system specifies the degree of emergency by averaging the scores of the variables. Due to the high importance of the questions and high difference between emergency patients and others, the averaging method could be used to decrease the complexity of the system.

\subsection{Module of herniated disc}

The module of herniated disc is activated due to pain in the leg and low back or in the arm and neck. Antecedents' variables of fuzzy rules of severity of pain in the leg/low back and arm/neck are shown in Figure 10. Figure 11 presents some of the rules and membership functions of variables of this module.

\subsection{Module of mechanical pain}

The module of mechanical pain is activated because of pain in the low back or pain in the neck. Antecedents' variables of fuzzy rules of severity of pain in the low back and neck are shown in Figure 12. Figure 13 shows some of the rules and membership functions of variables of this module.

\subsection{Modules of spinal stenosis}

The module of spinal stenosis is activated because of pain in either legs or both arms. Antecedents' variables of fuzzy rules of severity of pain in both legs and both arms are shown in Figure 14. Figure 15 presents some

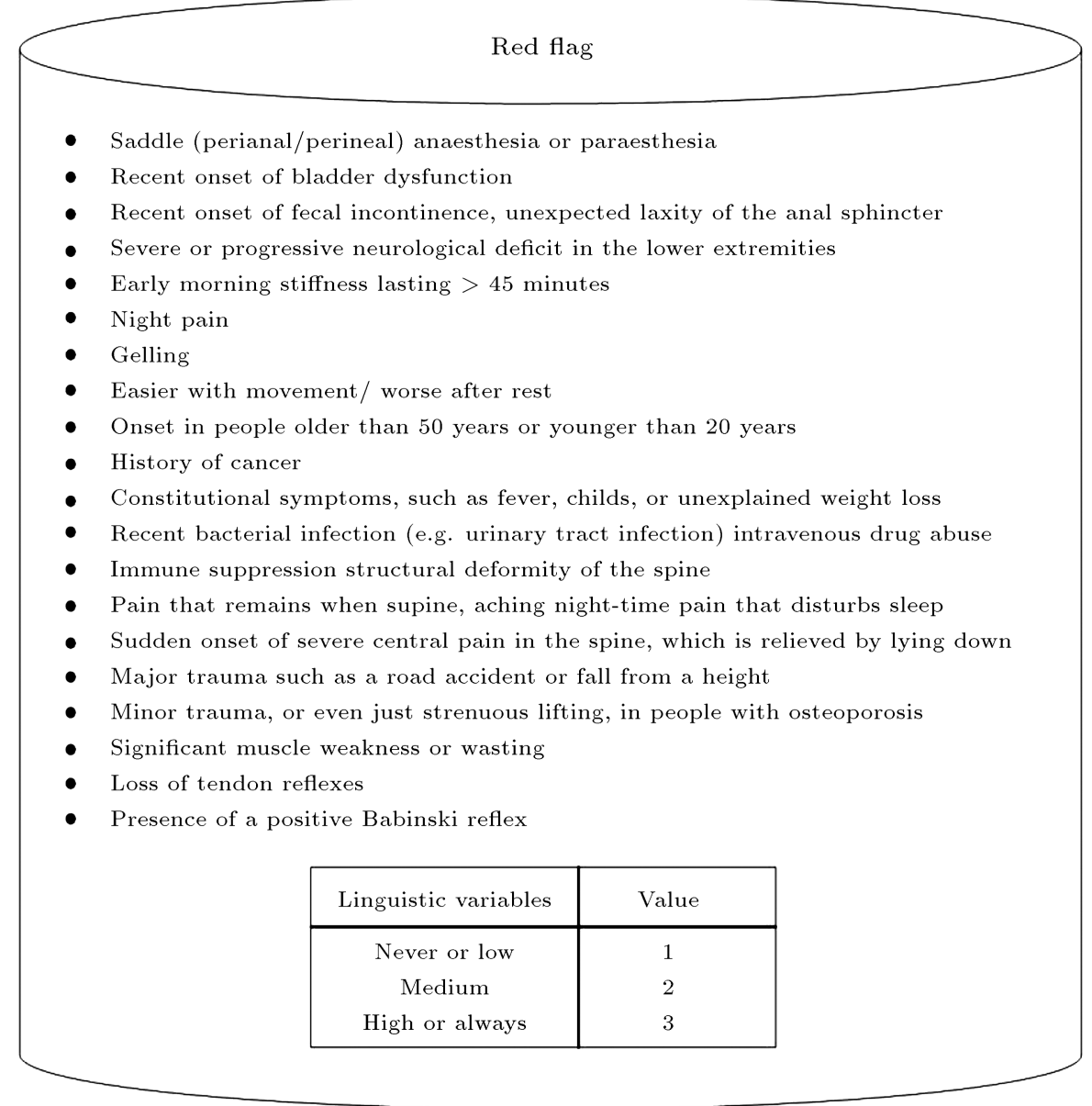

Figure 9. Antecedents of rules of module of Red Flag. 
Fuzzy variables of pain in leg and low back

- Local pain in the low back

- Pain in the legs, feet and toes

- Numbness in the legs, feet and toes

- Tingling in the legs, feet and toes

- Sciatica

- Muscle weakness in the thighs and calves

- Back stiffness or soreness

- Incontinence
Fuzzy variables of pain in arm and neck

- Local pain in the neck

- Pain in the arm, hands and toes

- Numbness in the arms and hands

- Tingling in the arms and hands

- Neck stiffness or soreness

- Traveling pain radiating along the nerve throughout the arm

- Muscle weakness in the shoulders, arms and elbows

Figure 10. Antecedents' variables of fuzzy rules of severity of pain in leg and low back/arm and neck.

Severity of pain Duration of pain Dependency of pain
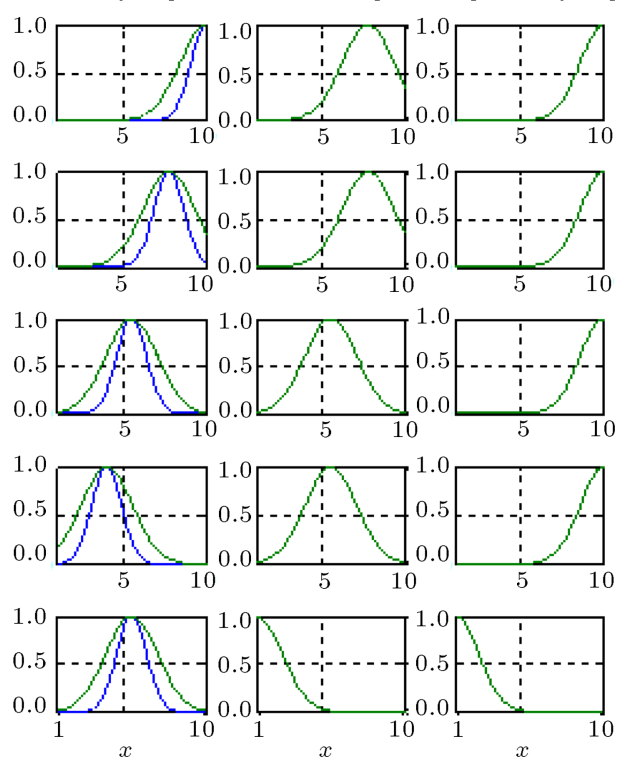

Herniated disc Mechanical pain Spinal stenosis
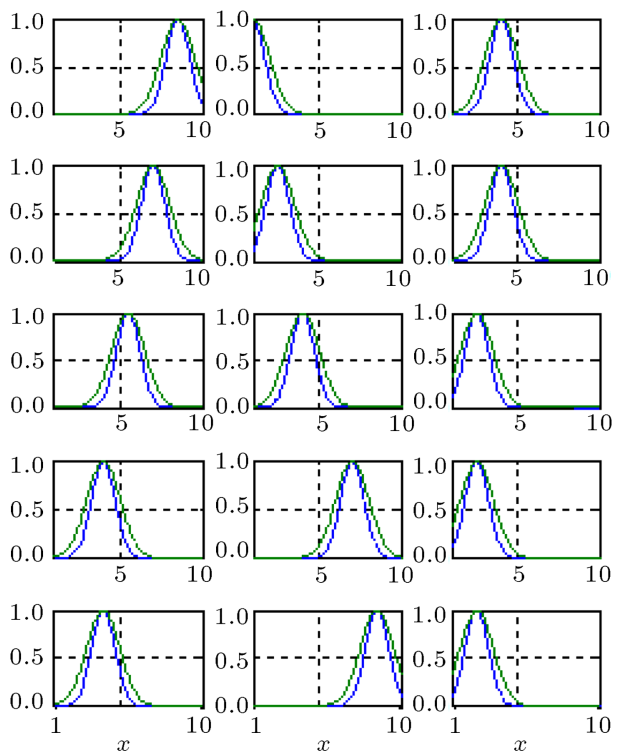

Figure 11. Schematic view of rules related to the module of herniated disc.

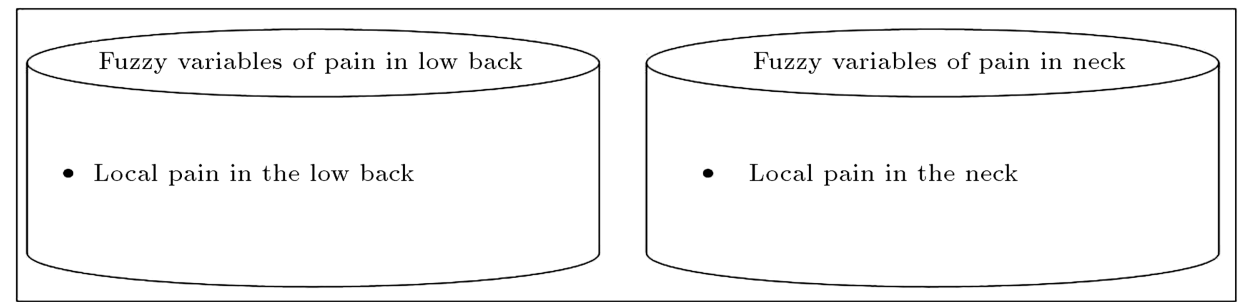

Figure 12. Antecedents' variables of fuzzy rules of severity of pain in low back/neck.

of the rules and membership functions of variables of this module.

\subsection{Module of nerve root}

The module of nerve root is activated to prove the herniated disc problem and find the exact location of the problem by investigating some clinical symptoms. The system could find the exact location of the problem between lumbar and cervical discs. The domain of the system in diagnosing the herniated disc is represented in Figure 16. To accelerate the search for the exact location of the disorder, the system asks some questions to investigate the symptoms based on prevalence of the disorder. These questions have a major role in finding the exact location and ensuring the patient's malingering. Variables of rules for the herniated disc in the low back and neck are represented in Figure 17.

\subsection{Module of scoliosis lordosis kyphosis}

The module of scoliosis lordosis kyphosis is activated to prove the mechanical disorder. Scoliosis, lordosis, kyphosis, and forward head are four problems that 
Severity of pain Duration of pain Dependency of pain
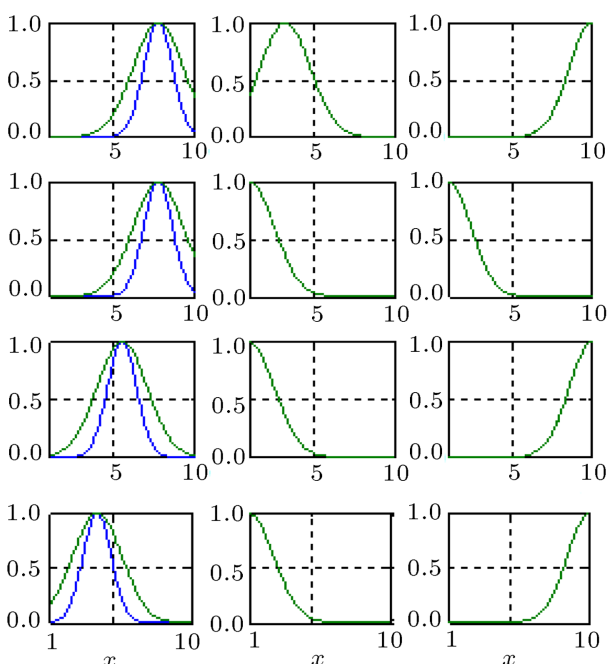

Herniated disc Mechanical pain Spinal stenosis
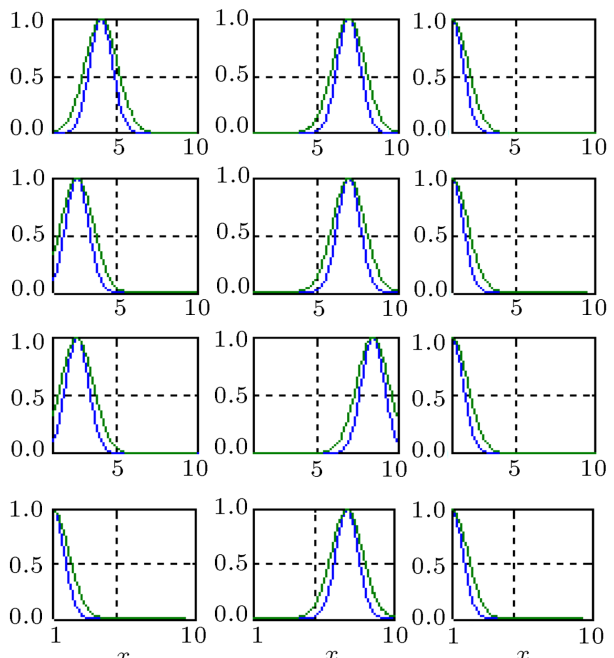

Figure 13. Schematic view of rules related to the module of mechanical pain.

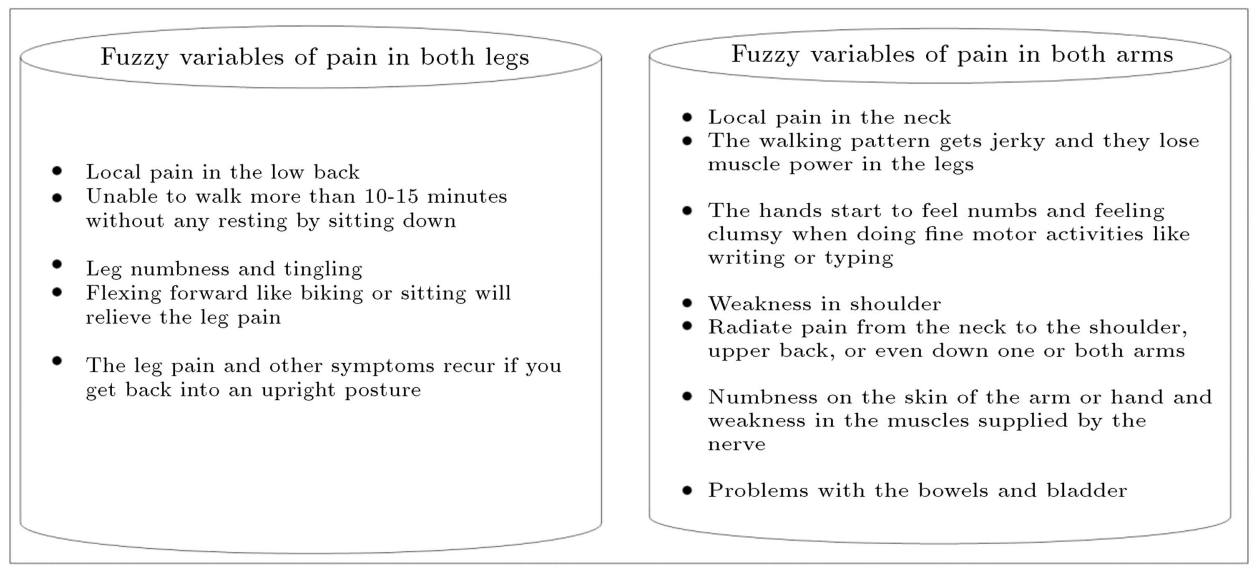

Figure 14. Antecedents' variables of fuzzy rules of severity of pain in both legs/arms.

Severity of pain Duration of pain Dependency of pain
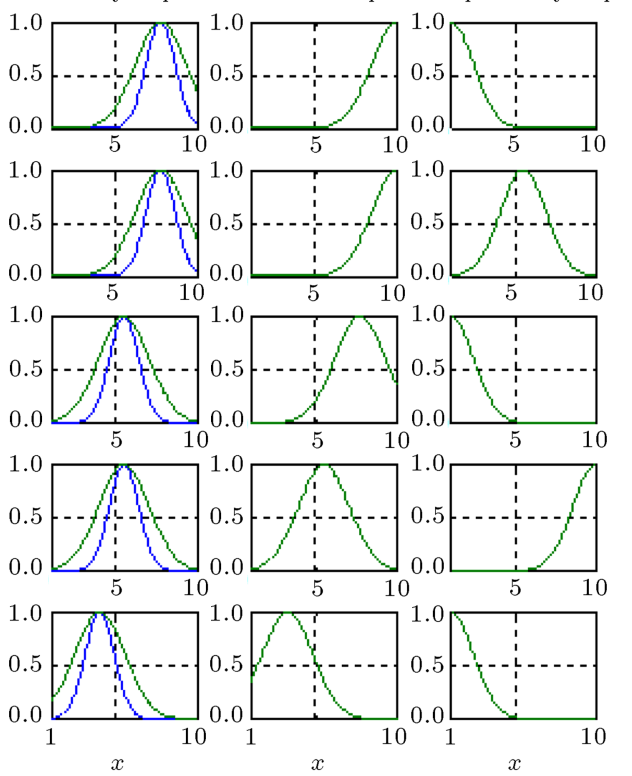

Herniated disc Mechanical pain Spinal stenosis
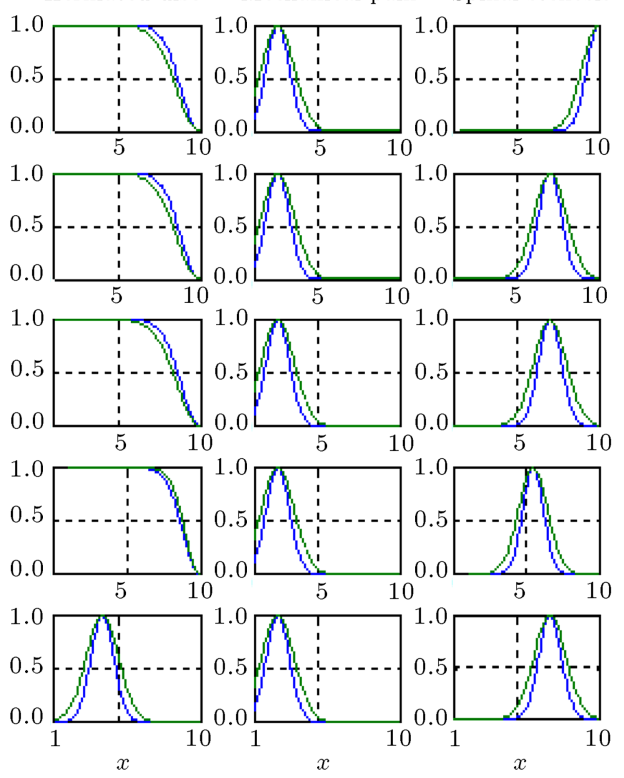

Figure 15. Schematic view of rules related to the module of spinal stenosis. 


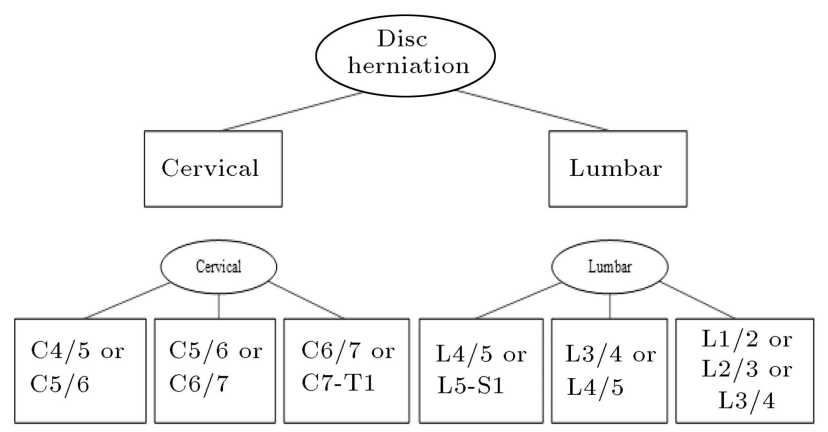

Figure 16. Domain of the system in diagnosing herniated disc.

have some overlaps with mechanical pain. To diagnose these disorders, the system investigates some of their symptoms to distinguish them from mechanical disorder. Antecedents of fuzzy rules of this module are represented in Figure 18.

\subsection{Module of vascular problem}

The module of vascular problem is activated to prove spinal stenosis disorder. Some of the symptoms are common to the vascular problems and spinal stenosis. To distinguish these disorders, the system investigates some of the uncommon symptoms of the vascular problem. Antecedents of fuzzy rules of this module are represented in Figure 18.

\subsection{Module of yellow flag and risk factors}

The aim of the psychosocial assessment is to find those patients who are likely to develop chronicity. The factors which highlight the patient's risk of chronicity can be identified using the 'yellow flag' system [37]. Risk factors increase the potential for back and neck problems and patients could decrease the pain by removing them. The factors of the yellow flag and risk factor are represented in Figure 19.

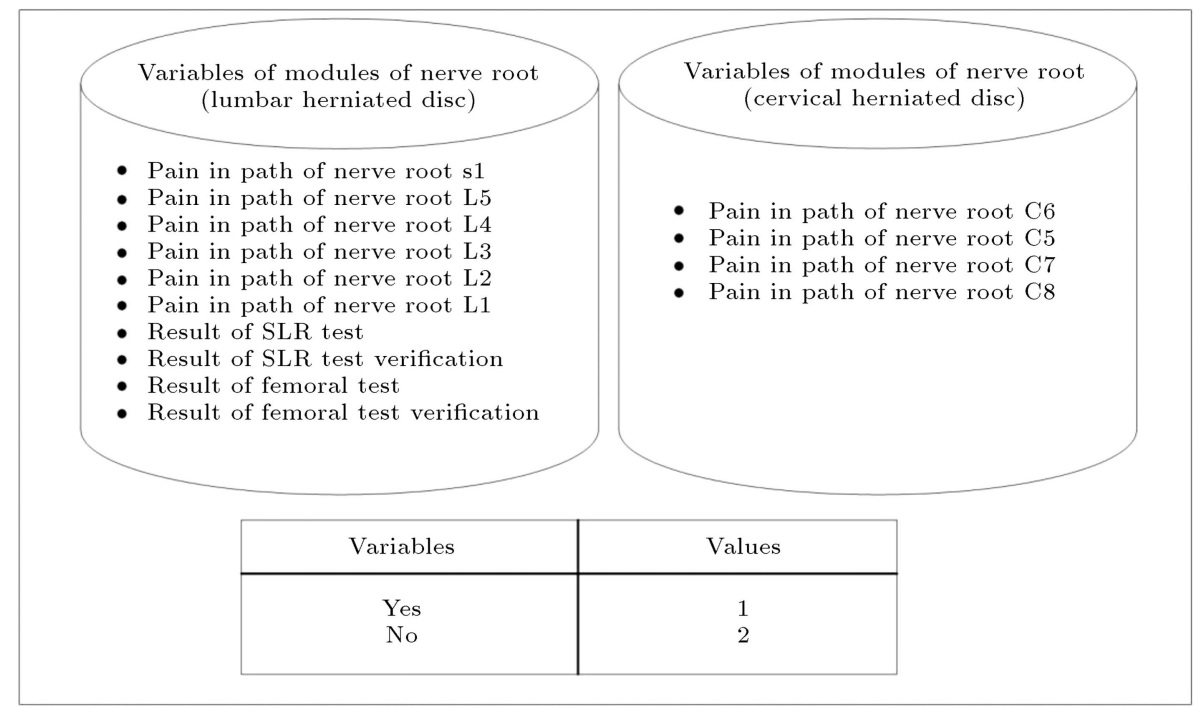

Figure 17. Antecedents, variables of fuzzy rules of module of nerve root.

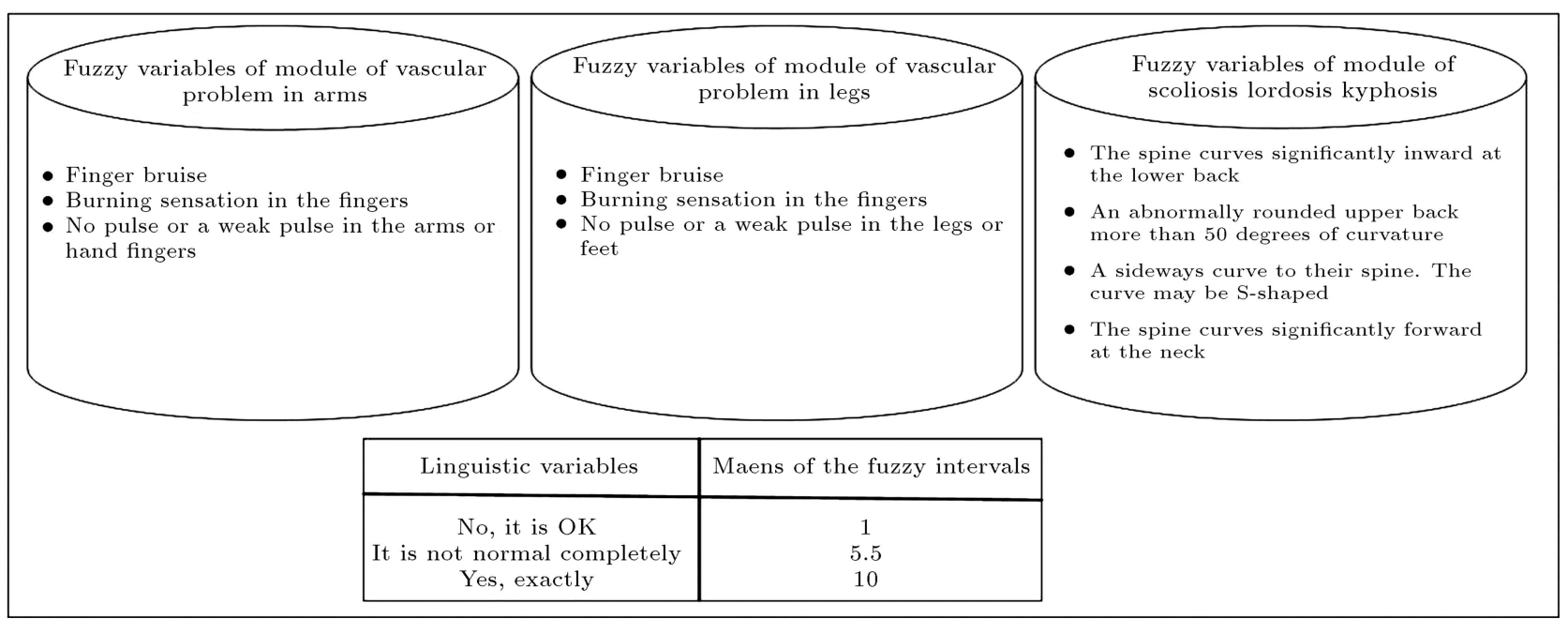

Figure 18. Antecedents of fuzzy rules of module of vascular problems/scoliosis lordosis kyphosis. 


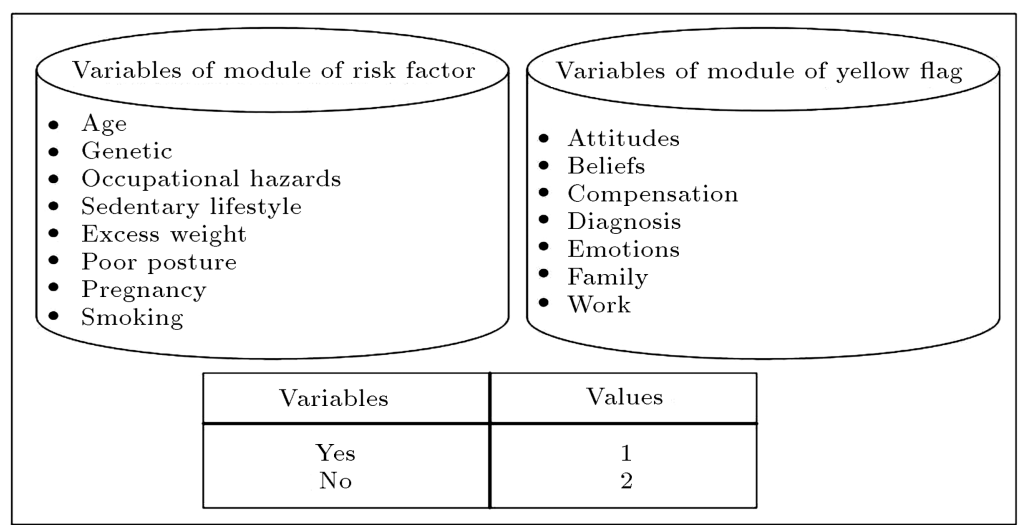

Figure 19. Antecedents of rules of module of yellow flag and risk factor.

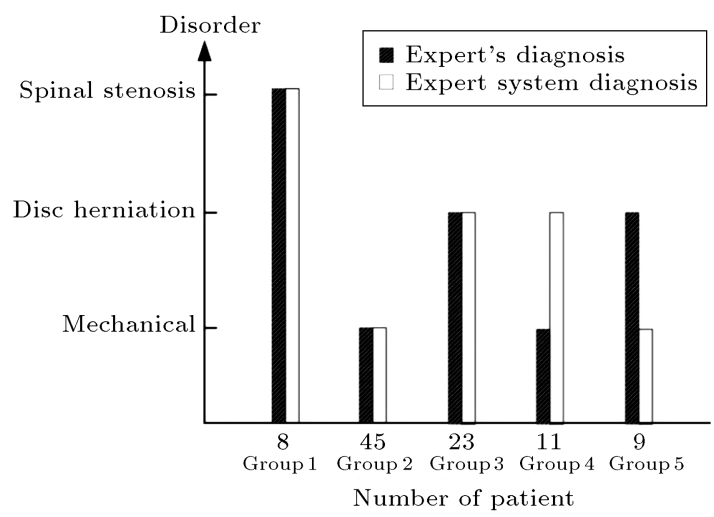

(a)

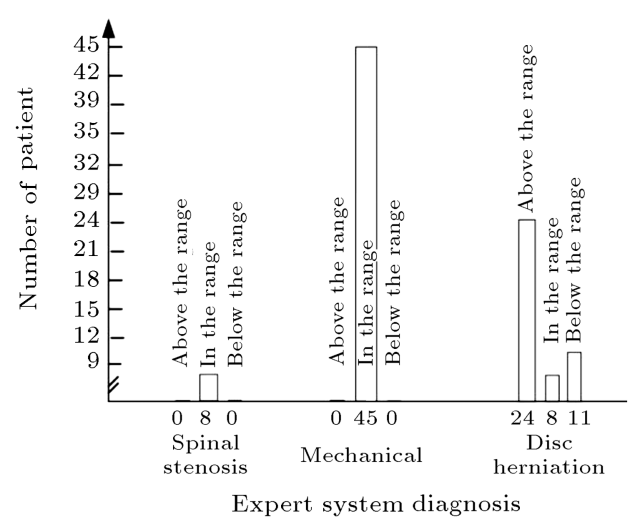

(c)

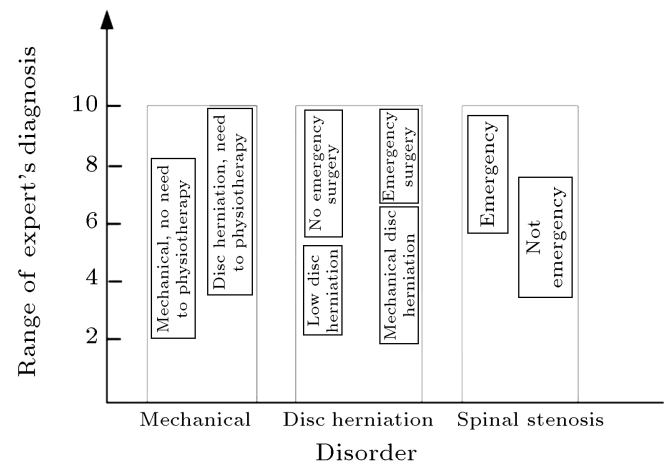

(b)

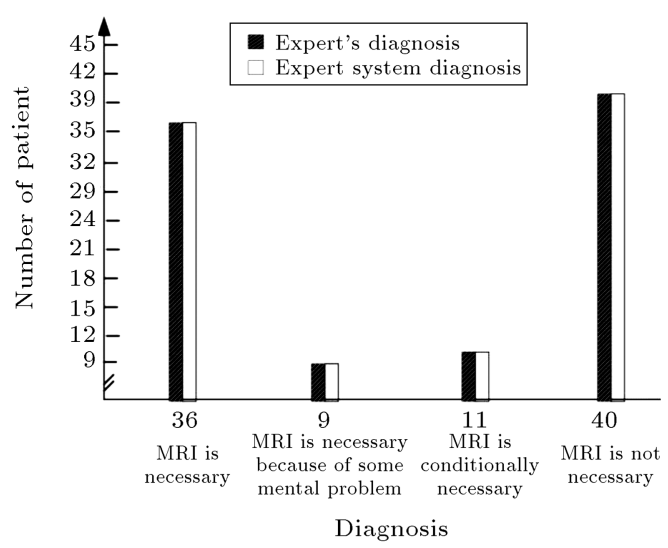

(d)

Figure 20. (a) Performance comparison of the expert and the system in primal diagnosis of problem. (b) Ranges of the expert diagnosis about the problem severity. (c) Performance comparison of the expert and the system in primal diagnosis of problem severity. (d) Performance comparison of the expert and the system in determination of necessity to MRI.

\section{Evaluating system performance}

The system consists of two stages: forward chaining for primal diagnosis and backward chaining for proving the primal diagnosis. The outputs of forward chaining stage are diagnosis of type of disorder and diagnosis of its severity. Declaring the necessity of providing MRI is the output of backward chaining stage. Each of the stages is tested separately with $25 \%$ of the patient's data. The results are as follows: one of the outputs of the forward chaining phase is diagnosis of the type of disorder between the three main disorders (herniated disc, mechanical pain, and spinal stenosis). For the comparison of the proposed system with the neurosurgeon in performing primal diagnosis of type of disorder, the expert system performance has been tested for 96 patients and the result is presented in Figure 20(a).

Following Figure 20(a), the diagnoses are categorized in five groups. As shown in Figure 20(a), the 
developed system's diagnoses and the neurosurgeon's diagnoses are completely equal in $79 \%$ of the data with 76 patients. The neurosurgeon's diagnoses of the disorders of patients of groups 4 and 5 are between herniated disc and mechanical pain. This is due to mechanical pain with low level of severity of the herniated disc disorder. The diagnosis of the developed system is mechanical pain or herniated disc in the first step. One of the other purposes of the developed system is diagnosing the severity of the problem. The expert's diagnosis is linguistic, so allocating an exact crisp value to the neurosurgeon's diagnosis is not feasible. The range for each diagnosis of the neurosurgeon is represented in Figure 20(b).

If the diagnosis of the developed system is in the range of the expert's diagnosis, the expert system performs properly. A comparison of the system's performance with the neurosurgeon in diagnosing the severity of the disorder is represented in Figure 20(c). Eighty-four patients are diagnosed properly and 11 diagnoses are below the range. All the 11 patients have herniated disc problem. The high overlapping between the herniated disc and mechanical pain results in this incompatibility.

Declaring the necessity of providing MRI is essential to complete the diagnosis. The necessity of providing MRI is categorized in four classes: MRI is necessary, MRI is necessary because of mental problems, MRI is conditionally necessary, and MRI is not necessary. A comparison of the developed system's performance with neurosurgeon in diagnosing the necessity of MRI is represented in Figure 20(d). The developed system is thoroughly successful in diagnosing the necessity for the patients. Accurate diagnosis of the disorder severity for the patients that need to take MRI is not feasible. As represented in Figure 20(d), all the patients diagnosed wrongly in previous steps are diagnosed properly in the final step.

\section{Discussion and conclusion}

The overlapping between the spinal cord disorders and the high uncertainty in some of the symptoms make diagnosis with computer programs complicated. On the other hand, the delay in diagnosing the disorders may increase the severity of pain and the cost of treatments. The proposed expert system in this paper alleviated these hazards and diagnosed between the nine spinal cord disorders, namely cervical herniated disc, lumbar herniated disc, mechanical pain, cervical spinal stenosis, lumbar spinal stenosis, scoliosis, lordosis, kyphosis, and forward head. The proposed system combined inference methods of forward and backward chaining. It could diagnose the type of disorder and its exact location by asking important questions about the patient's medical history and their clinical data.
By classifying the symptoms using different guidelines, type- 1 and type- 2 fuzzy logic systems were used and the severity of the pain was determined between 1 and 10. The modular structure of the knowledge base accelerated the diagnosis, and the proposed system after it could guess the location of the disorder without MR image processing, declared the need for taking MRI. One of the most important features of the proposed system was compatibility with a wide range of physicians by tuning its parameters. Moreover, the ability to update the parameters after each correct diagnosis made the system more robust.

In order to make a strong knowledge base, the data of 184 patients were used to extract the rules of the knowledge base. In the verification phase, the data of 96 patients were considered to define initial parameters and a validation test was done for them. Although the system could improve itself after each diagnosis, the future work can increase its performance by using the diagnoses of more neurosurgeons together to achieve a range for the parameters of the developed system. Another study that can be carried out is combining the developed system with image processing expert systems.

\section{References}

1. Andersson, G.B. "Epidemiological features of chronic low-back pain", The Lancet, 354(9178), pp. 581-585 (1999).

2. Koh, J., Chaudhary, V., Dhillon, G. "Disc herniation diagnosis in MRI using a CAD framework and a twolevel classifier", Int J Comput Assist Radiol Surg, 7(6), pp. 861-869 (2012).

3. Patel, V.L., Shortliffe, E.H., Stefanelli, M., Szolovits, P., Berthold, M.R., Bellazzi, R., and Abu-Hanna, A. "The coming of age of artificial intelligence in medicine", Artificial Intelligence in Medicine, 46(1), pp. 5-17 (2009).

4. Miller, P.L. "The evaluation of artificial intelligence systems in medicine", Comput Methods Programs Biomed, 22(1), pp. 5-11 (1986).

5. Seising, R. "From vagueness in medical thought to the foundations of fuzzy reasoning in medical diagnosis", Artif Intell Med, 38(3), pp. 237-256 (2006).

6. Bharti, P.K., Silawat, N., Singh, P.P., Singh, M.P., Shukla, M., Chand G., and Singh, N. "The usefulness of a new rapid diagnostic test, the first response malaria combo ( $\mathrm{pLDH} / \mathrm{HRP} 2$ ) card test, for malaria diagnosis in the forested belt of central India", Malar $J, 7$, p.126 (2008).

7. Obot, O.U. and Uzoka, F.M. "Experimental study of fuzzy-rule based management of tropical diseases: case of malaria diagnosis", Stud Health Technol Inform, 137(1), pp. 328-339 (2008).

8. Akinyokun, C.O., Obot, O.U., Uzoka, F.M., and Andy, J.J. "A neuro-fuzzy decision support system for the 
diagnosis of heart failure", Stud Health Technol Inform, 156(1), pp. 231-244 (2010).

9. Fazel Zaranid, M.H., Zolnoori, M., Moin, M., and Heidarnejad, H. "Fuzzy rule-based expert system for diagnosing asthma", Scientia Iranica, 17(2), pp. 129142 (2010).

10. Kadhim, M.A., Alam, M.A., and Kaur, H. "Design and implementation of fuzzy expert system for back pain diagnosis", Int Journal of Innovative Technology \& Creative Engineering, 1(9), pp. 16-22 (2011).

11. Sari, M., Gulbandilar, E., and Cimbiz, A. "Prediction of low back pain with two expert systems", J Med Sys, 36(3), pp. 1523-1527 (2012).

12. Esteban, B., Tejeda-Lorente, A., Porcel, C., Arroyo, M., and Herrera-Viedma, E. "TPLUFIB-WEB: A fuzzy linguistic Web system to help in the treatment of low back pain problems", Knowledge-Based Systems, 67(1), pp. 429-438 (2014).

13. Gulbandialr, E., Sari, M., and Cimbiz, A. "Prediction of low back pain using a fuzzy logic algorithm", American Journal of Biomedical Science and Engineering, 1(5), pp. 58-62 (2015).

14. Ohri, K., Singh, H., and Sharma, A. "Fuzzy expert system for diagnosis of breast cancer", Wireless Communications, Signal Processing and Networking (WiSPNET), International Conference on, Chennai, India, pp. 2487-2492 (2016).

15. Gal, N., Andrei D., Stoicu-Tivadar V., Nemeş D.I., and Nn̆dăşan E. "Fuzzy expert system prediction of lumbar spine subchondral sclerosis and lumbar disk hernia", Soft Computing Applications, pp. 2487-2492, Springer, Cham (2016).

16. Katigari, M.R., Ayatollahi, H., Malek, M., and Haghighi, M.K. "Fuzzy expert system for diagnosing diabetic neuropathy", World Journal of Diabetes, 8(2), p. 80 (2017)

17. Fazel Zarandi, M.H., Zarinbal, M., and Izadi, M. "Systematic image processing for diagnosing brain tumors: A type-II fuzzy expert system approach", Applied Soft Computing, 11(1), pp. 285-294 (2011).

18. Rahimi Damirchi-Darasi, S., Fazel Zarandi, M.H., and Izadi, M. "Type-2 fuzzy hybrid expert system for diagnosis of degenerative disc diseases", Modeling, Identification, Simulation \& Control (AIJ-MISC), 45(2), pp. 53-62 (2013).

19. Zarinbal, M., Turksen, L.B., Fazel Zarandi, M.H., and Izadi, M. "Interval type-2 fuzzy image processing expert system for diagnosing brain tumors", Norbert Wiener in the 21st Century (21CW), 2014 IEEE Conference on, Boston, MA, USA, pp. 1-8 (2014).

20. Zarinbal, M., Fazel Zarandi, M.H., Turksen, L.B., and Izadi, M. "A type-2 fuzzy image processing expert system for diagnosing brain tumors", Journal of Medical Systems, 39(10), p. 1 (2015).

21. Fazel Zarandi, M.H., Rahimi Damirchi-Darasi, S., Izadi, M., Turksen, I.B., and Arabzadeh Ghahazi, M.
"Fuzzy rule based expert system to diagnose spinal cord disorders", Norbert Wiener in the 21st Century (21CW), 2014 IEEE Conference on, Boston, MA, USA, pp. 1-5 (2014).

22. "Mechanical pain definition", Retrieved 1/15/2016, from:

http://www.spine-health.com/glossary/mechanicalpain

23. "Diseases and conditions herniated disk", Retrieved 1/15/2016, from http://www.mayoclinic.org/diseasesconditions/herniated-disk/basics/definition/c on-20029957 (2014).

24. "Diseases and conditions spinal stenosis", Retrieved 1/15/2016, from http://www.mayoclinic.org/diseasesconditions/spinal-stenosis/basics/definition/ con-20036105.

25. Revord, J.P. "Typical symptoms of a herniated disc", Retrieved 1/15/2016, from http://www.spinehealth.com/conditions/herniated-disc/typicalsymptoms-a-herniated-disc.

26. Staehler, R.A. "Cervical herniated disc symptoms and treatment options", Retrieved 1/15/2016, from http://www.spine-health.com/conditions/herniateddisc/cervical-herniated-disc-symptoms-andtreatment-options

27. Marieb, E.N. and Hoehn, K., Human Anatomy \& Physiology, Pearson Education (2007).

28. "Yellow flags in back pain", Retrieved 1/15/2016, from:

http://www.sheffieldbackpain.com/professionalresources/learning/in-detail/yellow-flags-in-back-pain.

29. Mendel, J.M. and John, R.I.B. "Type-2 fuzzy sets made simple", Fuzzy Systems, IEEE Transactions on, 10(2), pp. 117-127 (2002).

30. Mendel, J.M. "Advances in type-2 fuzzy sets and systems", Information Sciences, 177(1), pp. 84-110 (2007).

31. Emami, M.R., Türksen, I.B., and Goldenberg, A.A. "A unified parameterized formulation of reasoning in fuzzy modeling and control", Fuzzy Sets and Systems, 108(1), pp. 59-81 (1999).

32. Mendel, J., Hagras, H., Tan, W.-W., Melek, W.W., and Ying, H., Introduction to Type-2 Fuzzy Logic Control: Theory and Applications, John Wiley \& Sons (2014).

33. Karnik, N.N. and Mendel, J.M. "Introduction to type2 fuzzy logic systems", IEEE International Conference on Fuzzy Systems Proceedings. IEEE World Congress on Computational Intelligence (Cat. No. 98CH36228), Anchorage, AK, pp. 915-920 (1998).

34. Karnik, N.N., Mendel, J.M., and Qilian, L. "Type-2 fuzzy logic systems", Fuzzy Systems, IEEE Transactions on, 7(6), pp. 643-658 (1999).

35. Leekwijck, W.V. and Kerre, E.E. "Defuzzification: criteria and classification", Fuzzy Sets and Systems, 108(2), pp. 159-78 (1999). 
36. "Spinal disc problems (including red flag signs)", Retrieved 1/15/2016, from http://patient. info/doctor/ spinal-disc-problems-including-red-flag-signs.

37. Hochschuler, S.H. "Back pain risk factors: What can increase the potential for back problems?" Retrieved 1/15/2016, from http://www.spine-health. com/conditions/lower-back-pain/back-pain-riskfactors-

what-can-increase-potential-back-problems

38. Liang, Q. and Mendel, J.M. "Interval type-2 fuzzy logic systems: theory and design", Fuzzy Systems, IEEE Transactions on, 8(5), pp. 535-550 (2000)

\section{Biographies}

Saeede Rahimi Damirchi-Darasi received her BSc and MSc degrees in Industrial Engineering from Amirkabir University of Technology (AUT), Tehran, Iran, in 2012 and 2014, respectively. Her research interests are expert systems, artificial engineering, healthcare, and fuzzy logic systems.

Mohammad Hossein Fazel Zarandi is a Professor in the Department of Industrial Engineering at Amirkabir University of Technology, Tehran, Iran, and a member of the Knowledge-Information Systems Laboratory of the University of Toronto, Canada. His main research interests focus on intelligent information systems, soft computing, computational intelligence, fuzzy sets and systems, multi-agent systems, networks, meta-heuristics, and optimization. He has authored several books, scientific papers, and technical reports in the mentioned areas, most of which are accessible on the web. He has also taught several courses in fuzzy systems engineering, decision support systems, management information systems, artificial intelligence and expert systems, systems analysis and design, scheduling, neural networks, simulations, and production planning and control at several universities in Iran and North America.

Ismail Burhan Turksen received BSc and MSc degrees in Industrial Engineering and $\mathrm{PhD}$ degree in Systems Management and Operations Research from the University of Pittsburgh, USA. He became Full Professor at the University of Toronto, Canada, in 1983 and was appointed Head of the Department of Industrial Engineering at TOBB University of Economics and Technology. He is an active editorial board member of various journals, such as Fuzzy Sets and Systems, Approximate Reasoning, Decision Support Systems, Information Sciences, Expert Systems and its Applications, Journal of Advanced Computational Intelligence, Transactions on Operational Research, and Applied Soft Computing. Also, he is a fellow of IFSA and IEEE, and a member of IIE, CSIE, CORS, IFSA, NAFIPS, APEO, APET, TORS, ACM, etc.

Mina Izadi is a highly experienced neurosurgeon that cooperates with Professor Fazel Zarandi in development of healthcare expert systems associated with nervous system. 